\title{
Are young men who overestimate drinking by others more likely to respond to an electronic normative feedback brief intervention for unhealthy alcohol use?
}

\author{
Nicolas Bertholet, Jean-Bernard Daeppen, John A. Cunningham, Bernard Burnand, \\ Gerhard Gmel, Jacques Gaume
}

Nicolas Bertholet, MD, MSc, Privat-Docent, Senior Lecturer, Associate Physician, Alcohol Treatment Center, Department of community medicine and health, Lausanne University Hospital, Lausanne, Switzerland

Jean-Bernard Daeppen, MD, Professor, Alcohol Treatment Center, Department of community medicine and health, Lausanne University Hospital, Lausanne, Switzerland

John A. Cunningham, PhD, Professor, National Institute for Mental Health Research, Australian National University, Canberra, Australia and Centre for Addiction and Mental Health, Toronto, Canada

Bernard Burnand, MD, MPH, Professor, Institute of social and preventive medicine, Lausanne University Hospital, Lausanne, Switzerland

Gerhard Gmel, PhD, Associate Professor, Alcohol Treatment Center, Department of community medicine and health, Lausanne University Hospital, Lausanne, Switzerland

Jacques Gaume, PhD, Alcohol Treatment Center, Department of community medicine and health, Lausanne University Hospital, Lausanne, Switzerland

Word count: 2695

\section{Randomized trial registration:}

The trial was registered at current controlled trials: ISRCTN55991918

\section{Corresponding author:}

Nicolas Bertholet, MD, MSc

Beaumont 21b, P2, 02

1011 Lausanne, CHUV

Nicolas.Bertholet@chuv.ch 


\section{ABSTRACT}

Aim: To tested whether the efficacy of an internet-based brief intervention that included normative drinking feedback varied with estimations of the drinking of others.

Methods: This study is a secondary analysis of a randomized controlled trial showing an intervention effect on weekly drinking. Participants were males with unhealthy alcohol use (mean age $[S D]=20.8$ [1.1]). Before the trial, participants were asked to estimate the percentage of men their age who drink more than they do. Using their self-reported drinking data, the "perceived" percentage of people their age and gender who drink more than they do, and data from Swiss statistics, we classified participants as overestimating $(>+10 \%)$, accurately $(-10 \%$ to $+10 \%)$ or underestimating $(<-10 \%)$ drinking by others.

Results: Of 734 participants with complete data, 427 overestimated, 205 accurately estimated and 102 underestimated the drinking of others. The mean (SD) number of drinks per week was 9.8 (7.9) and AUDIT score was 10.6 (4.2). In stratified negative binomial regression models predicting drinks per week, at 6 months, and controlling for baseline drinks per week, the intervention was effective among those overestimating (IRR $[95 \% \mathrm{Cl}]=0.86[0.74 ; 0.98])$, but showed no effect among those accurately estimating $(\operatorname{IRR}[95 \% \mathrm{Cl}]=0.83[0.66 ; 1.03])$ or underestimating IRR $[95 \% \mathrm{Cl}]=1.21[0.92 ; 1.60])$ the drinking of others.

Conclusions: Perception of drinking by others appears to be a moderator of effect of an electronic feedback intervention among hazardous drinkers. This finding is consistent with the hypothesis that correcting the perceptions of others' drinking is a potential mechanism of action in normative feedback paradigms. 


\section{INTRODUCTION}

Unhealthy alcohol use is a major public health problem (Rehm, 2011) and has been the target of multiple secondary prevention brief intervention models (Babor et al., 2010). It is one of the leading modifiable risk factors of morbidity and mortality in young adults (Marmet, Rehm, Gmel, Frick, \& Gmel, 2014). Over the past decade, there has been a large increase in the development of web-based brief interventions for unhealthy alcohol use. These interventions are able to reach a broad population of users who are not necessarily seeking treatment (Cunningham \& Breslin, 2004), and they have multiple advantages including low cost, less burden on primary care providers, no requirement for extensive training, continuous access for participants, no geographical restrictions, and reduced fear of stigma since the interventions are anonymous (Lapham et al., 2012). Recent systematic reviews indicate that this method of delivery of brief intervention has potential efficacy, but underline that few studies have investigated nonstudent populations, even though electronic interventions show promise for young adults and adolescents (Donoghue, Patton, Phillips, Deluca, \& Drummond, 2014; Khadjesari, Murray, Hewitt, Hartley, \& Godfrey, 2011; Patton et al., 2014; Riper et al., 2014; Riper et al., 2011; Rooke, Thorsteinsson, Karpin, Copeland, \& Allsop, 2010).

Normative feedback has often been included as a component of brief web-based interventions for unhealthy alcohol use and is effective in reducing alcohol use (Cunningham, Hendershot, Murphy, \& Neighbors, 2012; Cunningham, Wild, Cordingley, van Mierlo, \& Humphreys, 2009; Kypri et al., 2013; Lewis \& Neighbors, 2007; Neighbors 
et al., 2010). Intervention effects are significant, though small (Foxcroft, Moreira, Almeida Santimano, \& Smith, 2015). In general, normative feedback aims at highlighting discrepancies between one's perception of others' drinking and one's actual alcohol use by using specific feedback (Lewis, Neighbors, Oster-Aaland, Kirkeby, \& Larimer, 2007). For individuals with unhealthy use, the aim is reduced consumption through normative feedback. The hypothesized mechanism of action of normative feedback relies on the following assumptions: 1) individuals with unhealthy alcohol use overestimate the drinking by others (i.e. they perceive heavy drinking as the norm, or they misperceive their own drinking compared to the drinking of others); 2) these misperceptions can be modified; and 3) providing current norms will correct misperceptions and lead to reduced drinking (Borsari \& Carey, 2001; Prentice \& Miller, 1993). There is preliminary evidence supporting the theoretical hypothesis of normative feedback, namely, that changes in drinking have been shown to be mediated by changes in perceived norms following the feedback (Neighbors, Larimer, \& Lewis, 2004). Following this hypothesis, the normative feedback is an active ingredient and the change in perceived norms the mechanism of change (i.e. the normative feedback introduces or increases discrepancy between one's perception and actual norms).

As with other components of brief interventions, the study of mechanisms of action of normative feedback could lead to the development of more effective interventions (Miller et al., 2013) and to a better understanding of the potential or inherent risks of the intervention. Some concerns have been raised about possible "boomerang" effects of normative feedback, but Prince et al. (Prince, Reid, Carey, \& Neighbors, 2014) investigated the impact of normative feedback among light drinkers in four samples and 
found no evidence of increased drinking following normative feedback. Nevertheless, while the perception of others' drinking is often exaggerated among young adults (Baer \& Carney, 1993; Borsari \& Carey, 2003), not all individuals with unhealthy alcohol use overestimate drinking by others. In two studies conducted in a sample of Swiss young men, some unhealthy alcohol users correctly estimated their own drinking, relative to peer drinking, or even underestimated drinking by others; up to $20 \%$ of individuals who drank 15 or more drinks per week accurately estimated or underestimated drinking by others (Bertholet, Faouzi, Studer, Daeppen, \& Gmel, 2013; Bertholet, Gaume, Faouzi, Daeppen, \& Gmel, 2011). Therefore, when delivered to large samples of unhealthy alcohol users, interventions that include normative feedback elements are likely to reach individuals who correctly perceive the norms and understand that their own consumption is relatively high. Determining whether normative feedback effects differ according to perceptions of how much others drink is important. Individuals who overestimate drinking by others should benefit most from normative feedback, but iatrogenic or boomerang effects are possible for those individuals who underestimate.

Therefore, the aim of this study was to determine whether the perception of drinking by others improves the efficacy of brief interventions which include normative feedback as a component, i.e., do perceptions of drinking act as a moderator of outcome effects? Using data from a randomized controlled trial of web-based brief interventions that showed a significant intervention effect on drinking at 6 months, we investigated the impact these perceptions had on the intervention effect. We hypothesized that there would be greater reductions in alcohol use among those who overestimated the drinking of others. 


\section{MATERIAL AND METHODS:}

This study is a secondary analysis of a two-group, parallel randomized controlled trial showing an intervention effect on weekly drinking among 737 Swiss males with unhealthy alcohol use having a mean age (SD) of 20.8 (1.1) years (Bertholet et al., 2015). Participants were recruited in Switzerland within a population-based study of young males, the Cohort on Substance Use Risk Factors (CSURF, see http://www.csurf.ch).

Unhealthy alcohol use was defined as $>14$ drinks/week or $>=6$ drinks/occasion at least monthly or Alcohol Use Disorders Identification Test (AUDIT) scores $>=8$. Participants were randomized to receive an internet-based intervention $(n=367)$ or no treatment $(n=370)$ and were followed at one month and at six months. The intervention had a positive effect on the primary outcome (number of drinks per week at 6 months). Details of that research have been reported elsewhere (Bertholet et al., 2015). First, at the cohort study assessment that took place before the recruitment in the randomized trial, the participants were asked to estimate the percentage of people their age and gender who drink more than them with the question: In your opinion, what is the percentage of people your age and gender drinking more than you do. At the randomized trial baseline assessment, participants were then asked to report their weekly drinking and frequency of binge drinking (defined as six or more drinks per occasion), through questions about typical drinking frequency and amount consumed per typical drinking day. These quantity and frequency measures have been validated (Rehm, 1998). Number of drinks per week was calculated by multiplying number of drinking days per week by number of standard drinks per drinking days. This assessment was repeated at 
the six-month follow up. Reported drinking at baseline was compared to national data from the Swiss Health Survey (OFS, 2004) and shown to intervention group participants in the form of normative feedback, showing the percentage of individuals of the same age and gender who drink as much as, and less than they do. These comparisons were also presented in pie charts. Other intervention elements consisted of feedback on consequences of drinking, amount of calories consumed in alcoholic drinks over the past 12 months, estimated blood alcohol content during episodes of heavy drinking, recommendations for low-risk drinking, and general information on alcohol and health. Using self-reported baseline drinking, participants' perceptions of peer drinking, and the national data used to provide the normative feedback, we classified participants as overestimating $(>+10 \%)$, accurately $(-10 \%$ to $+10 \%)$ or underestimating $(<-10 \%)$ drinking by others Participants with complete reported and perceived drinking data $(n=734)$ were used in the present study.

We chose to focus on number of drinks per week as an outcome variable because it was the primary outcome in the main study where an intervention effect was found. Analyses: First, we compared each demographic and baseline drinking variable (number of drinks per week, binge drinking prevalence and AUDIT scores) between the three perception of drinking groups using Kruskal-Wallis for continuous variables and Chi square for categorical variables. Analyses were stratified by perception of drinking across three groups of participants who overestimated, accurately estimated, and underestimated drinking by others. Negative binomial regression models controlling for baseline drinking predicted weekly drinking at six months. 


\section{RESULTS:}

Of the 734 participants with complete data, 427 (58.2\%) overestimated, 205 (27.9\%) accurately estimated and 102 (13.9\%) underestimated drinking by others. Baseline characteristics of participants are presented in Table 1. Mean (SD) number of drinks per week $=9.8(7.9)$ and mean AUDIT score $=10.6(4.2)$. Number of drinks per week, binge drinking and AUDIT scores all differed significantly between participants overestimating, accurately estimating or underestimating drinking by others. Over estimators reported higher weekly drinking, binge drinking prevalence and AUDIT scores.

Table 2 show the results of the negative binomial regression models stratified by perception of drinking of others and Figure 1 show graphical representations of these effects. The intervention had a significant effect on mean number of drinks per week at 6 months among participants overestimating the drinking of others (incidence risk ratio $(\mathrm{IRR})=0.86,95 \% \mathrm{Cl}: 0.74-0.98, \mathrm{p}=0.03)$. Consistently, participants in the intervention group decreased their drinking more importantly than those in the control group in this sub-group on Figure 1 (thick lines).

For participants accurately estimating drinking by others, there was a trend for a positive intervention effect. Graphs showed more important decrease among participants in the intervention group (medium lines), however, this effect was not significant at a standard alpha level of $0.05(\mathrm{IRR}=0.83,95 \% \mathrm{Cl}: 0.66-1.03, \mathrm{p}=0.10)$. Among those underestimating peer drinking, the graphs showed a trend for the controls to decrease their weekly drinking more than did those receiving the intervention (thin 
lines), which is in line with the IRR larger than 1 (IRR=1.21) even if this result was not statistically significant $(95 \% \mathrm{Cl}: 0.92-1.60, \mathrm{p}=0.16)$.

\section{DISCUSSION:}

We investigated whether the perceptions of peer drinking in a web-based brief intervention with normative feedback moderated the effects on weekly drinking in young men with unhealthy alcohol use. The intervention was effective among hazardous young male drinkers who overestimated, but had no significant effect on those who accurately (or who underestimated) the drinking of others. Among over estimators, the intervention group reported significantly less drinking than did the controls six months later. No statistically significant differences were found among those who accurately estimated or underestimated peer drinking. These results are consistent with the hypothesis that correcting misperceptions about others' drinking is a potential mechanism of action of normative feedback (Neighbors et al., 2004). In our study, we showed that misperception was a moderator of a web-based brief intervention with normative feedback, indicating that intervention was mostly effective with those individuals who overestimate drinking by others. This finding adds to the current literature which showed that changes in perceived norms mediated the efficacy of normative feedback interventions delivered face-to-face or in computerized format (Carey, Henson, Carey, \& Maisto, 2010; Lewis \& Neighbors, 2007; Wood, Capone, Laforge, Erickson, \& Brand, 2007). As the design of the study was developed to be minimal (e.g. short questionnaires) to facilitate young men participation, we were not able to capture changes in perceived norms and thus test mediation. 
Questions remain as to whether normative feedback can be safely administered to all individuals having unhealthy alcohol use. Indeed, in contrast to those overestimating or accurately estimating drinking by others, under estimators randomized to a control condition tended to decrease their alcohol use more than did those randomized to receive normative feedback. Even though the difference between groups was not significant, these results might suggest that this small group (13.9\%) of hazardous drinkers who are underestimating peer drinking may negatively respond to normative feedback. Considering it was the smallest group, there was limited power to detect significant effects. These results remain consistent with the hypothesized mechanism of action of normative feedback. If highlighting discrepancies between norms and current drinking can lead to decreased drinking among individuals who are overestimating the norms, one could alternatively hypothesize that providing normative feedback to individuals who already believe they drink more than their peers might lead to unexpected and potentially unfavorable outcomes. Our results may be seen as different in this respect from what has been shown previously for light drinkers, where boomerang effects have not been observed (Prince et al., 2014). Even in the absence of boomerang effects among light drinkers, normative feedback may have a different impact on unhealthy alcohol users who believe that only a small portion of their peers drink more than they do, when in reality, the proportion is much larger. In other words, individuals having unhealthy alcohol use who misperceive their behavior as rare may negatively respond (i.e., increase their drinking) after learning through normative feedback that other individuals their age and gender are drinking even more than they 
are. Our results call for future research that focuses on mechanisms of action in webbased interventions and proactively manipulates those potential active mechanisms. The present study has several limitations. The study was not designed with these analyses in mind and therefore the study sample was not determined to test these secondary hypotheses. Therefore we had limited statistical power, especially to assess the intervention effect among the limited number of participants who underestimated drinking by others. Also, the assessments were based solely on self-reports of drinking and were therefore prone to social desirability and recall bias. Perception of peer drinking was assessed with a generic question that did not differentiate between drinking patterns and was not reassessed following the intervention, thus we were not able to determine whether any changes in perception were mediating the intervention effect. Also, in our study, we used national descriptive norms. Acting on perceived drinking of friends, or perceived drinking of local peers may produce larger effects than acting on perceived drinking of peers on a national level (Carey et al., 2010). Incorporating local drinking norms in the feedback may increase the intervention effect. Nevertheless, providing feedback on local norms or drinking norms among friends could be challenging in an internet-based intervention. In addition, the results might very well be due to some unknown factor that co-varies with the perception of peer drinking. It is important to take into account the intervention mode of delivery. For example, the present study assessed the effects of normative feedback within the context of a web-based intervention; these results may not generalize to face-to-face normative feedback interventions, where other factors (such as counselor behaviors) might play a role in intervention effects. It should finally be 
noted that while the intervention contained normative feedback, it was not limited to this element, thus other components of the intervention may have influenced the outcome. These elements were not considered in this analysis as the design of the original study was minimal (e.g. very short questionnaires) to facilitate young men participation.

Despite these limitations, the present study has some notable strengths. We examined mechanisms operating in an effective intervention (i.e., effects on the primary outcome variable in the original study, number of drinks per week (Bertholet et al., 2015)). The design allowed testing of the moderating effect of drinking perceptions, and was conducted in a population-based sample of young males. This research was not limited to college or university students, who have customarily been the main focus of webbased brief interventions. Also, we were able to analyze a particular mechanism hypothesized to affect drinking. Even if there was some imprecision in matching the drinking of young males to the national norms, the data used in the present analyses were the actual data which generated the normative feedback on which participants compared themselves.

\section{CONCLUSIONS:}

In summary, our results are consistent with the theoretical hypothesis underlying the normative feedback approach, but also contain potential caveats regarding who might benefit (or possibly be adversely affected) by receiving the intervention. Further research is needed to characterize and delineate how particular subgroups in the larger population will respond to normative feedback brief interventions that are web-based. 
In terms of clinical outcomes, we believe that the observed changes in drinking in a nontreatment seeking general population for an intervention with minimal costs and large scalability is significant: for those who overestimated drinking of others, we compare a decrease of 2 drinks per week in the intervention group to a decrease of 0.3 drinks in the controls. Looking at IRR, there was $14 \%$ less alcohol use in the intervention compared to the control group among the over estimators $(1.00-0.86=0.14)$. 
TABLE 1: baseline characteristics of study participants

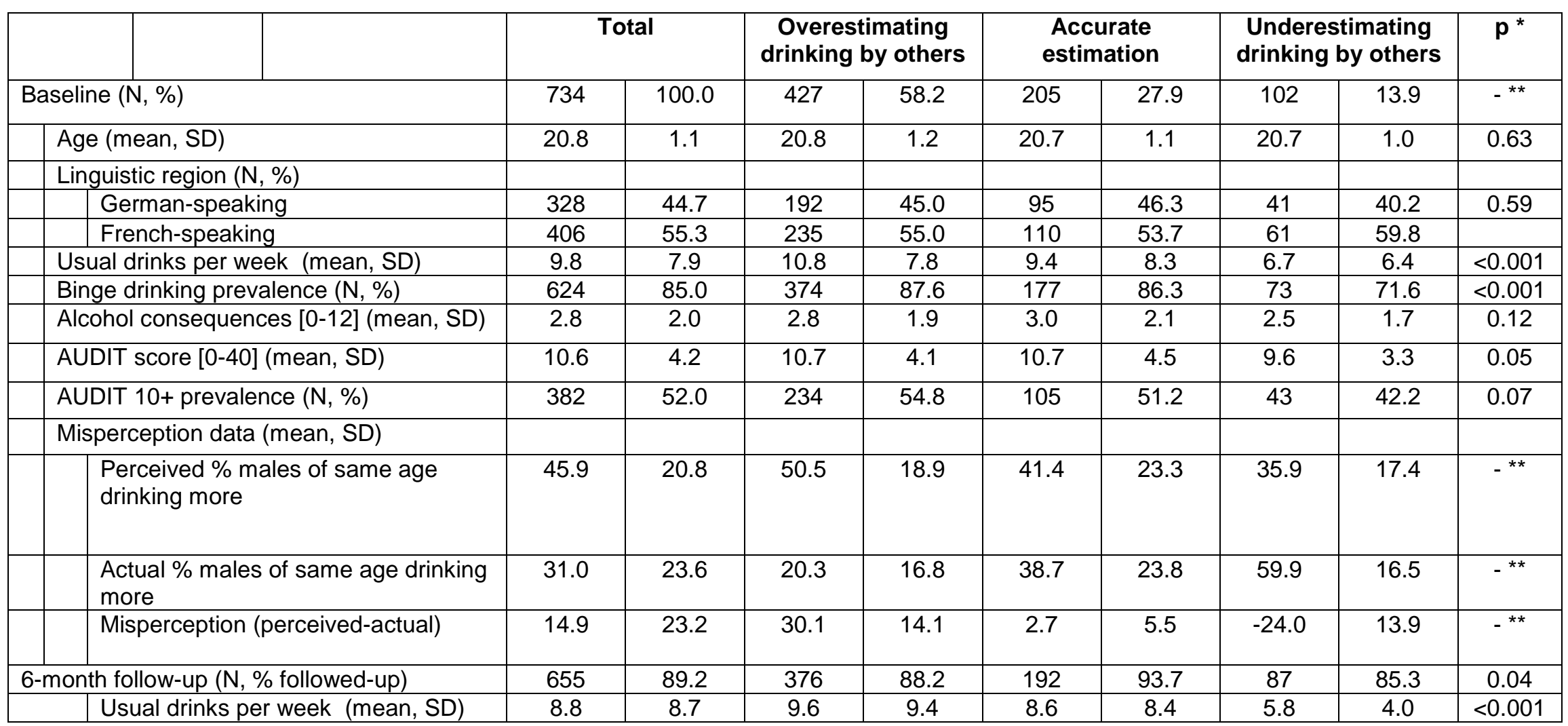

* $p$ values for tests comparing the 3 levels of perception (Kruskal-Wallis for continuous variables and Chi square for categorical variables).

** Not calculated as different by design. 
TABLE 2: intervention effects on the number of drinks per week at 6 months, stratified by perception of drinking

\begin{tabular}{|l|c|c|c|}
\hline & IRR (drinks per week) & $\mathbf{9 5 \%}$ ( & P \\
\hline OVERESTIMATING DRINKING BY OTHERS & & & 0.03 \\
\hline Intervention & 0.86 & $0.74 ; 0.98$ & $<0.001$ \\
\hline Baseline drinking & 1.05 & $1.04 ; 1.07$ & \\
\hline $\begin{array}{l}\text { ACCURATELY ESTIMATING DRINKING BY } \\
\text { OTHERS }\end{array}$ & & & 0.10 \\
\hline Intervention & 0.83 & $0.66 ; 1.03$ & $<0.001$ \\
\hline Baseline drinking & 1.06 & $1.05 ; 1.08$ & \\
\hline UNDERESTIMATING DRINKING BY OTHERS & & & 0.16 \\
\hline Intervention & 1.21 & $0.92 ; 1.60$ & 0.001 \\
\hline Baseline drinking & 1.04 & $1.01 ; 1.06$ & \\
\hline
\end{tabular}


FIGURE 1: Number of drinks per week at baseline and 6 months, by study group, stratified by perception of drinking by others

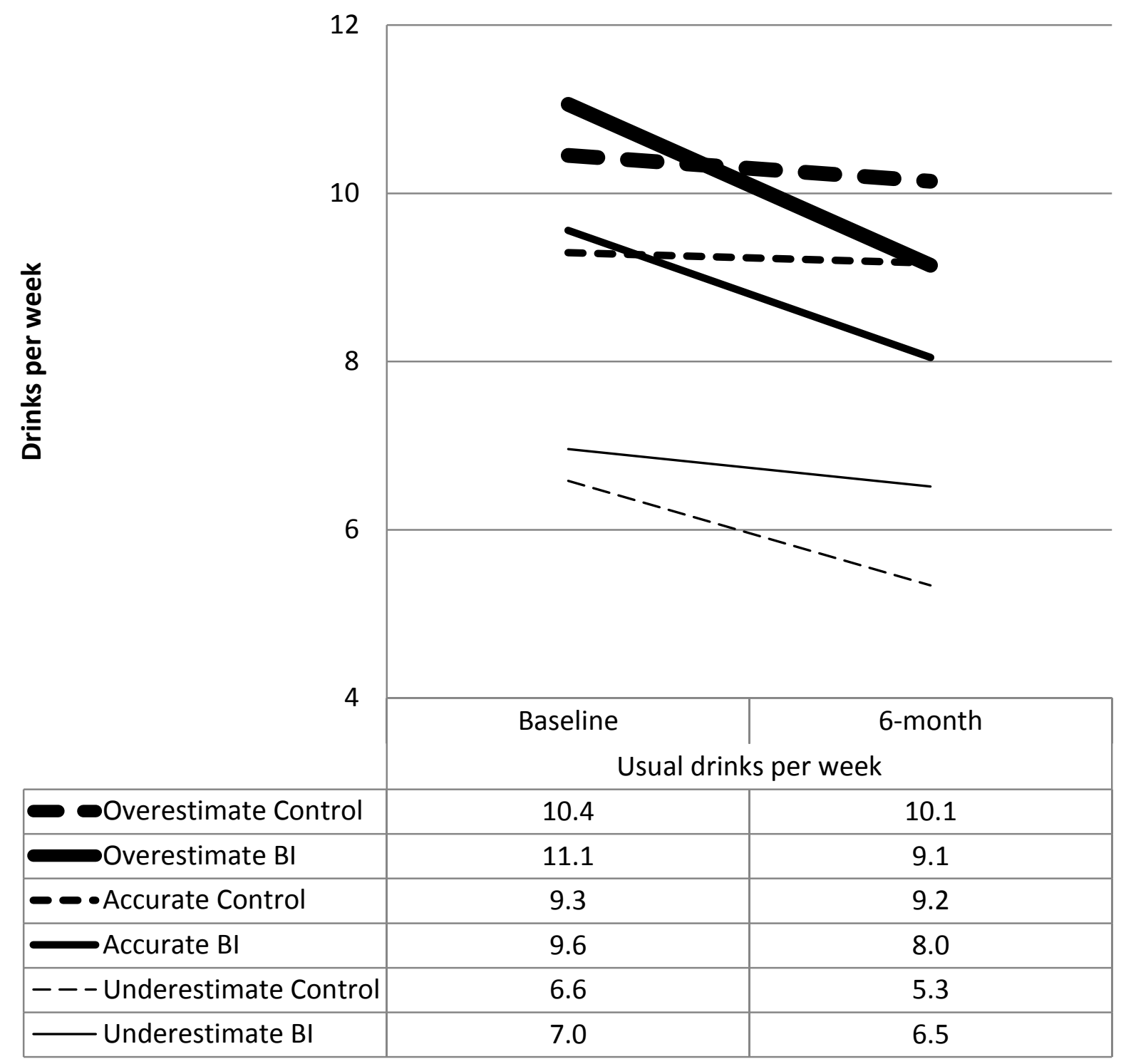




\section{Funding and trial registration:}

The study was funded by the Swiss National Science Foundation (grant 325130_135538/1, PI: N Bertholet).

C-SURF was funded by the Swiss National Science Foundation (33CSCO-122679, PI:

G Gmel).

Researchers were independent from the Swiss National Science Foundation. The Swiss National Science Foundation had no role in the study design, data collection, data analysis, data interpretation or writing of the report.

\section{Ethics approval:}

The study was approved by the Ethics Committee for Clinical Research of the Lausanne University Medical School. C-SURF was approved by the Ethics Committee for Clinical Research of the Lausanne University Medical School.

\section{Authorship/contributorship:}

$\mathrm{NB}, \mathrm{JBD}, \mathrm{JC}, \mathrm{BB}$ and $\mathrm{GG}$ designed the randomized trial and the current study. GG is the primary investigator of C-SURF. NB, JBD and BB conducted the randomized trial. $\mathrm{NB}, \mathrm{JG}$ and $\mathrm{JC}$ designed the analyses presented herein. JG and NB conducted the analyses. All authors interpreted the study results. NB wrote the first draft of the manuscript. All authors edited the paper and contributed significant intellectual content to the manuscript. 


\section{REFERENCES}

Babor, T. F., Caetano, R., Casswell, S., Edwards, G., Giesbrecht, N., Graham, K., . . Rossow, I. (2010). Alcohol: No Ordinary Commodity. Research and Public Policy. New York: Oxford University Press.

Baer, J. S., \& Carney, M. M. (1993). Biases in the perceptions of the consequences of alcohol use among college students. J Stud Alcohol, 54(1), 54-60.

Bertholet, N., Cunningham, J. A., Faouzi, M., Gaume, J., Gmel, G., Burnand, B., \& Daeppen, J. B. (2015). Internet-based brief intervention for young men with unhealthy alcohol use: a randomized controlled trial in a general population sample. Addiction, 110(11), 1735-1743. doi: 10.1111/add.13051

Bertholet, N., Faouzi, M., Studer, J., Daeppen, J. B., \& Gmel, G. (2013). Perception of tobacco, cannabis, and alcohol use of others is associated with one's own use. Addict Sci Clin Pract, 8(1), 15. doi: 10.1186/1940-0640-8-15

Bertholet, N., Gaume, J., Faouzi, M., Daeppen, J. B., \& Gmel, G. (2011). Perception of the amount of drinking by others in a sample of 20-year-old men: the more I think you drink, the more I drink. Alcohol Alcohol, 46(1), 83-87. doi: 10.1093/alcalc/agq084

Borsari, B., \& Carey, K. B. (2001). Peer influences on college drinking: a review of the research. J Subst Abuse, 13(4), 391-424.

Borsari, B., \& Carey, K. B. (2003). Descriptive and injunctive norms in college drinking: a meta-analytic integration. J Stud Alcohol, 64(3), 331-341.

Carey, K. B., Henson, J. M., Carey, M. P., \& Maisto, S. A. (2010). Perceived Norms Mediate Effects of a Brief Motivational Intervention for Sanctioned College Drinkers. Clin Psychol (New York), 17(1), 58-71. doi: 10.1111/j.14682850.2009.01194.x

Cunningham, J. A., \& Breslin, F. C. (2004). Only one in three people with alcohol abuse or dependence ever seek treatment. Addict Behav, 29, 221-223.

Cunningham, J. A., Hendershot, C. S., Murphy, M., \& Neighbors, C. (2012). Pragmatic randomized controlled trial of providing access to a brief personalized alcohol feedback intervention in university students. Addict Sci Clin Pract, 7(1), 21. doi: 10.1186/1940-0640-7-21

Cunningham, J. A., Wild, T. C., Cordingley, J., van Mierlo, T., \& Humphreys, K. (2009). A randomized controlled trial of an internet-based brief intervention for alcohol abusers. Addiction, 12(104), 2023-2032.

Donoghue, K., Patton, R., Phillips, T., Deluca, P., \& Drummond, C. (2014). The effectiveness of electronic screening and brief intervention for reducing levels of alcohol consumption: a systematic review and meta-analysis. J Med Internet Res, 16(6), e142. doi: 10.2196/jmir.3193

Foxcroft, D. R., Moreira, M. T., Almeida Santimano, N. M., \& Smith, L. A. (2015). Social norms information for alcohol misuse in university and college students. Cochrane Database Syst Rev, 1, CD006748. doi: 10.1002/14651858.CD006748.pub3

Khadjesari, Z., Murray, E., Hewitt, C., Hartley, S., \& Godfrey, C. (2011). Can standalone computer-based interventions reduce alcohol consumption? A systematic review. Addiction, 106(2), 267-282. doi: 10.1111/j.1360-0443.2010.03214.x 
Kypri, K., McCambridge, J., Vater, T., Bowe, S. J., Saunders, J. B., Cunningham, J. A., \& Horton, N. J. (2013). Web-based alcohol intervention for Maori university students: double-blind, multi-site randomized controlled trial. Addiction, 108(2), 331-338. doi: 10.1111/j.1360-0443.2012.04067.x

Lapham, G. T., Hawkins, E. J., Chavez, L. J., Achtmeyer, C. E., Williams, E. C., Thomas, R. M., ... Bradley, K. A. (2012). Feedback from recently returned veterans on an anonymous web-based brief alcohol intervention. Addict Sci Clin Pract, 7(1), 17. doi: 10.1186/1940-0640-7-17

Lewis, M. A., \& Neighbors, C. (2007). Optimizing personalized normative feedback: the use of gender-specific referents. J Stud Alcohol Drugs, 68(2), 228-237.

Lewis, M. A., Neighbors, C., Oster-Aaland, L., Kirkeby, B. S., \& Larimer, M. E. (2007). Indicated prevention for incoming freshmen: personalized normative feedback and high-risk drinking. Addict Behav, 32(11), 2495-2508. doi: 10.1016/j.addbeh.2007.06.019

Marmet, S., Rehm, J., Gmel, G., Frick, H., \& Gmel, G. (2014). Alcohol-attributable mortality in Switzerland in 2011 - age-specific causes of death and impact of heavy versus non-heavy drinking. Swiss Med Wkly, 144, w13947. doi: $10.4414 / \mathrm{smw} .2014 .13947$

Miller, M. B., Leffingwell, T., Claborn, K., Meier, E., Walters, S., \& Neighbors, C. (2013). Personalized feedback interventions for college alcohol misuse: an update of Walters \& Neighbors (2005). Psychol Addict Behav, 27(4), 909-920. doi: 10.1037/a0031174

Neighbors, C., Larimer, M. E., \& Lewis, M. A. (2004). Targeting misperceptions of descriptive drinking norms: efficacy of a computer-delivered personalized normative feedback intervention. J Consult Clin Psychol, 72(3), 434-447. doi: 10.1037/0022-006X.72.3.434

Neighbors, C., Lewis, M. A., Atkins, D. C., Jensen, M. M., Walter, T., Fossos, N., . . . Larimer, M. E. (2010). Efficacy of web-based personalized normative feedback: a two-year randomized controlled trial. J Consult Clin Psychol, 78(6), 898-911. doi: 10.1037/a0020766

OFS. (2004). Enquête suisse sur la santé 2002. In O. f. d. I. statistique (Ed.), Statistique suisse. Neuchâtel: Office fédéral de la statistique.

Patton, R., Deluca, P., Kaner, E., Newbury-Birch, D., Phillips, T., \& Drummond, C. (2014). Alcohol screening and brief intervention for adolescents: the how, what and where of reducing alcohol consumption and related harm among young people. Alcohol Alcohol, 49(2), 207-212. doi: 10.1093/alcalc/agt165

Prentice, D. A., \& Miller, D. T. (1993). Pluralistic ignorance and alcohol use on campus: some consequences of misperceiving the social norm. J Pers Soc Psychol, 64(2), 243-256.

Prince, M. A., Reid, A., Carey, K. B., \& Neighbors, C. (2014). Effects of normative feedback for drinkers who consume less than the norm: Dodging the boomerang. Psychol Addict Behav, 28(2), 538-544. doi: 10.1037/a0036402

Rehm, J. (1998). Measuring quantity, frequency, and volume of drinking. Alcohol Clin Exp Res, 22(2 Suppl), 4S-14S.

Rehm, J. (2011). The risks associated with alcohol use and alcoholism. Alcohol Res Health, 34(2), 135-143. 
Riper, H., Blankers, M., Hadiwijaya, H., Cunningham, J., Clarke, S., Wiers, R., . . . Cuijpers, P. (2014). Effectiveness of guided and unguided low-intensity internet interventions for adult alcohol misuse: a meta-analysis. PloS one, 9(6), e99912. doi: 10.1371/journal.pone.0099912

Riper, H., Spek, V., Boon, B., Conijn, B., Kramer, J., Martin-Abello, K., \& Smit, F. (2011). Effectiveness of E-self-help interventions for curbing adult problem drinking: a meta-analysis. J Med Internet Res, 13(2), e42. doi: 10.2196/jmir.1691

Rooke, S., Thorsteinsson, E., Karpin, A., Copeland, J., \& Allsop, D. (2010). Computerdelivered interventions for alcohol and tobacco use: a meta-analysis. Addiction, 105(8), 1381-1390.

Wood, M. D., Capone, C., Laforge, R., Erickson, D. J., \& Brand, N. H. (2007). Brief motivational intervention and alcohol expectancy challenge with heavy drinking college students: a randomized factorial study. Addict Behav, 32(11), 2509-2528. doi: 10.1016/j.addbeh.2007.06.018 